OPEN ACCESS

Edited by:

Filippo Tempia,

University of Turin, Italy

Reviewed by:

Masoud Tahmasian,

Shahid Beheshti University, Iran

Xuetao Mu,

Chinese People's Armed Police General Hospital, China

${ }^{*}$ Correspondence: Kuncheng $\mathrm{Li}$

likuncheng@xwh.ccmu.edu.cn

Zhiqun Wang

wangzhiqun@126.com

tThese authors have contributed equally to this work.

Received: 21 December 2016 Accepted: 29 April 2017 Published: 16 May 2017

Citation:

Zheng W, Liu X, Song H, Li K and Wang Z (2017) Altered Functional Connectivity of Cognitive-Related Cerebellar Subregions in Alzheimer's Disease.

Front. Aging Neurosci. 9:143. doi: 10.3389/fnagi.2017.00143

\section{Altered Functional Connectivity of Cognitive-Related Cerebellar Subregions in Alzheimer's Disease}

\author{
Weimin Zheng ${ }^{1 \dagger}$, Xingyun Liu ${ }^{1 \dagger}$, Haiqing Song ${ }^{2}$, Kuncheng $\mathrm{Li}^{3,4 *}$ and Zhiqun Wang ${ }^{1 *}$ \\ ${ }^{1}$ Department of Radiology, Dongfang Hospital, Beijing University of Chinese Medicine, Beijing, China, ${ }^{2}$ Department of \\ Neurology, Xuanwu Hospital of Capital Medical University, Beijing, China, ${ }^{3}$ Department of Radiology, Xuanwu Hospital of \\ Capital Medical University, Beijing, China, ${ }^{4}$ Beijing Key Laboratory of Magnetic Resonance Imaging and Brain Informatics, \\ Beijing, China
}

Alzheimer's disease (AD) is the most common cause of dementia. Previous studies have found disrupted resting state functional connectivities (rsFCs) in various brain networks in the AD patients. However, few studies have focused on the rsFCs of the cerebellum and its sub-regions in the AD patients. In this study, we collected resting-state functional magnetic resonance imaging ( $\mathrm{rs}-\mathrm{fMRI}$ ) data including $32 \mathrm{AD}$ patients and 38 healthy controls (HCs). We selected two cognitive-related subregions of the cerebellum as seed region and mapped the whole-brain rsFCs for each subregion. We identified several distinct rsFC patterns of the two cognitive-related cerebellar subregions: default-mode network (DMN), frontoparietal network (FPN), visual network (VN) and sensorimotor network (SMN). Compared with the controls, the AD patients showed disrupted rsFCs in several different networks (DMN, VN and SMN), predicting the impairment of the functional integration in the cerebellum. Notably, these abnormal rsFCs of the two cerebellar subregions were closely associated with cognitive performance. Collectively, we demonstrated the distinct rsFCs patterns of cerebellar sub-regions with various functional networks, which were differentially impaired in the AD patients.

Keywords: Alzheimer's disease, resting state fMRI, functional connectivity, cerebellum, subregions, neuroimaging

\section{INTRODUCTION}

Alzheimer's disease (AD) is the most common cause of dementia manifested as progressive memory deficits and cognitive impairment (Seeley et al., 2009; Pievani et al., 2011a), which may be caused by the deposition of amyloid- $\beta$ plaques, neurofibrillary tangles and neuronal loss (Braak and Braak, 1991; Kandimalla et al., 2011, 2014). These AD pathological changes attack the cognitive related specific regions and disrupt the connectivity among these regions, which leads to the cognitive decline. Therefore, AD is regarded as a disconnection syndrome (Buckner et al., 2009). However, the mechanism is not very clear at present.

Resting-state functional magnetic resonance imaging (rs-fMRI) has been increasingly utilized to explore neural networks by investigating the brain low frequency fluctuations in the blood-oxygen level-dependent (BOLD) signals (Biswal et al., 1995; Zhang and Raichle, 2010). By using fMRI technique, previous $\mathrm{AD}$ studies have demonstrated that the disrupted resting-state functional connectivities (rsFCs) mainly concentrated on several key regions of neuronal degeneration in $\mathrm{AD}$, such as the anterior hippocampus (BA 27/28; Wang et al., 2006), posterior cingulate cortex (BA 23/29/31; Greicius et al., 2004; Zhang et al., 2010), prefrontal cortex (BA 8/9/10; 
Wang et al., 2007; Dai et al., 2012), inferior parietal lobe (BA 39/40; Wang et al., 2015) and thalamus (Wang et al., 2012). Besides these regions, cerebellum is recently regarded as an important cognitive-related region in $\mathrm{AD}$. The cerebellum has traditionally been considered as a reference region in $\mathrm{AD}$ since it has been reported to provide unbiased estimations for intensity normalization (Mevel et al., 2007; Chételat et al., 2008). However, the idea that the cerebellum remains unaffected by $\mathrm{AD}$ is challenged by several recent studies. For example, gray matter (GM) atrophy of the cerebellum was detected in $\mathrm{AD}$ in several neuroimaging studies (Thomann et al., 2008; Bas et al., 2009). In addition, the AD patients exhibited significantly lower cerebral blood flow (CBF; Hauser et al., 2013), abnormal activation during Stroop tests (Kaufmann et al., 2008) and impaired rsFCs of the cerebellum (Wang et al., 2007; Zhang et al., 2014) in several studies. Furthermore, the AD pathological changes have now been revealed in the cerebellum including deposits of amyloid- $\beta$ plaques, neurofibrillary tangles, increased microglia and so on (Larner, 1997; Ciavardelli et al., 2010; Sepulveda-Falla et al., 2011). These studies raised the interest of cerebellum which may be a vulnerable region in the $\mathrm{AD}$ patients. Thus, it is extremely important to ascertain the intrinsic rsFCs pattern of the cerebellum in AD.

The cerebellum is a region characterized with complex structure and function. Anatomically, the cerebellum is linked to the cerebrum through three cerebellar peduncles. It has efferent and afferent fibers between the vermis, hypothalamus and limbic system (Strata, 2015). Functionally, based on the anatomical links, the cerebellum is connected to several brain networks, which is involved in multimodal functions such as cognitive, emotional and sensorimotor processing (Schmahmann et al., 2007; Stoodley and Schmahmann, 2009; O'Reilly et al., 2010). Interestingly, several recent fMRI studies found the consistent rsFCs between the cerebellar sub-regions (lobule IX and Crus II) and the cognitive-related network regions. However, the results are not very consistent. Habas et al. (2009) reported lobule IX was linked with default mode network (DMN). However, Krienen and Buckner (2009) emphasized on Crus II and regarded it as an important role in DMN. Other studies from Sang et al. (2012) and Li et al. (2013) found the Crus II was typically connected with frontoparietal network (FPN) and the lobule IX was mainly connected with the DMN. Therefore, we sought to extend prior work to determine actual connectivity patterns of the cerebellar cognitive related subregions including the lobule IX and Crus II.

Considering the heterogeneity of the cerebellum and its association with $\mathrm{DMN}$, it is extremely important to ascertain the intrinsic rsFCs of the cerebellar cognitive-related subregions in AD. Until now, only a few previous resting state studies focused on cerebellar connectivity in the early stage of $\mathrm{AD}$ patients (Bai et al., 2011; Castellazzi et al., 2014). One study applied independent component analysis (ICA) to analyze the cerebellum-related networks in the $\mathrm{AD}$ patients (Castellazzi et al., 2014). Although ICA can decompose BOLD data into independent components using statistical method, there are still some challenges for the ICA method. For example, there were no priori criteria to identify the number of independent components in BOLD data, which contributed to the final results in a high degree (Fox and Raichle, 2007). Another rs-fMRI study explored regional cerebellar activation and rsFC in early risk state of $\mathrm{AD}$ (Bai et al., 2011). However, the study only selected part of lobule IX as seed region to detect the cerebellar connectivity. Furthermore, the BOLD data analysis was based on the $1.5 \mathrm{~T}$ MRI which might mask some subtle changes in the results. To the present, no studies have reported altered patterns of intrinsic cerebellum subregional connectivity (Crus II and lobule IX) in $\mathrm{AD}$ using 3T MRI.

Here, we aim to investigate the intrinsic rsFCs of the cerebellar cognitive related subregions in the $\mathrm{AD}$ patients by using rs-fMRI, and to explore the association between the abnormal cerebellar rsFCs changes and patients' cognitive performances. In order to explain that the results were not caused by the regional GM atrophy, we performed voxel-based morphometry (VBM) analysis and used the GM as nuisance covariate in the study. We hypothesized that the $\mathrm{AD}$ patients would show differentially disrupted rsFCs of cerebellar sub-regions, which were independent of GM atrophy and closely associated with cognitive performances.

\section{MATERIALS AND METHODS}

\section{Subjects}

Seventy-five subjects including $34 \mathrm{AD}$ patients and 41 age and gender matched healthy controls (HCs) were recruited and participated in the study at the memory clinic of Xuanwu Hospital. All the subjects were right-handed. The study was approved by the Medical Research Ethics Committee of Xuanwu Hospital. All subjects provided written informed consent prior to participation, consistent with the Declaration of Helsinki. The details of the consent form included the study's aim, inclusion and exclusion criteria, procedures, potential harm and benefits, medical care, privacy rights, and withdrawal process. They were informed of their right to discontinue participation at any time. During the preprocessing, we excluded five subjects (two AD patients and three HCs). Table 1 showed the clinical information for the remaining 70 participants, including $32 \mathrm{AD}$ patients and $38 \mathrm{HCs}$. All the $\mathrm{AD}$ patients underwent a complete physical and neurological examination standard laboratory tests, and an extensive battery of neuropsychological assessments, which included mini-mental state examination (MMSE), Clinical Dementia Rating (CDR), World Health Organization-University of California-Los Angeles Auditory Verbal Learning Test (WHO-UCLA-AVLT), the Extended Scale for Dementia (ESD), Montreal Cognitive Assessment (MoCA), Clock Drawing Task (CDT), Activity of Daily Living Scale (ADL), Functional Activities Questionary (FAQ), Hamilton Depression Scale (HAMD) and Hachinski Ischemic Score (HIS). The diagnosis of $\mathrm{AD}$ fulfilled the new research criteria for possible or probable AD (Dubois et al., 2007, 2010) 
TABLE 1 | Demographic and neuropsychological test.

\begin{tabular}{|c|c|c|c|}
\hline & $\mathrm{AD}(n=32)$ & HC $(n=38)$ & $P$ Value \\
\hline Age (years) & $52-86(71.25 \pm 8.63)$ & $50-86(68.39 \pm 7.78)$ & $0.15^{\mathrm{a}}$ \\
\hline Gender (male/female) & $14 / 18$ & $13 / 25$ & $0.41^{b}$ \\
\hline CDR & $0.5(n=14), 1(n=18)$ & 0 & - \\
\hline MMSE & $10-25(18.56 \pm 3.99)$ & $28-30(28.63 \pm 0.67)$ & $<0.001^{a}$ \\
\hline AVLT & $8-24(14.81 \pm 4.12)$ & $39-52(44.42 \pm 2.74)$ & $<0.001^{\mathrm{a}}$ \\
\hline ESD & $107-200(155.33 \pm 26.48)$ & $180-248(227.74 \pm 15.68)$ & $<0.001^{\mathrm{a}}$ \\
\hline MoCA & $8-19(14.94 \pm 3.23)$ & $27-30(28.63 \pm 0.67)$ & $<0.001^{a}$ \\
\hline CDT & $3-8(6.13 \pm 1.43)$ & $8-9(8.71 \pm 0.46)$ & $<0.001^{a}$ \\
\hline ADL & $22-45(30.41 \pm 7.21)$ & $20-22(21.08 \pm 0.78)$ & $<0.001^{\mathrm{a}}$ \\
\hline FAQ & $4-11(6.25 \pm 1.70)$ & $0-2(0.55 \pm 0.76)$ & $<0.001^{a}$ \\
\hline HAMD & $0-3(1.06 \pm 1.08)$ & $0-3(0.61 \pm 1.00)$ & $0.07^{\mathrm{a}}$ \\
\hline HIS & $0-3(1.16 \pm 0.77)$ & $0-3(1.13 \pm 1.07)$ & $0.91^{\mathrm{a}}$ \\
\hline
\end{tabular}

Data are presented as the range of minimum-maximum (mean $\pm S D$ ). Abbreviations: AD, Alzheimer's disease; ADL, Activity of Daily Living Scale; WHO-UCLA-AVLT, World Health Organization-University of California-Los Angeles Auditory Verbal Learning Test; CDR, Clinical Dementia Rating; CDT, Clock Drawing Task; ESD, The Extended Scale for Dementia; FAQ, Functional Activities Questionary; HAMD, Hamilton Depression Scale; HCs, healthy controls; HIS, Hachinski Ischemic Score; MMSE, Mini-Mental State Examination; MoCA, Montreal Cognitive Assessment. ${ }^{a}$ The $P$ value was obtained by two-sample two-tailed t-test; ${ }^{b}$ The $P$ value was obtained by two-tailed Pearson chi-square test.

The criteria for HCs were as follows: (a) no neurological or psychiatric disorders such as stroke, depression, epilepsy; (b) no neurological deficiencies such as visual or hearing loss; (c) no significant abnormal findings such as infarction or tumor in conventional brain MR imaging; (d) no cognitive complaints; (e) MMSE score of 28 or higher; and (f) CDR score of 0 .

\section{Data Acquisition}

MRI data were collected using a SIEMENS Trio 3-Tesla scanner (Siemens, Erlangen, Germany). Sponge pads were placed to limit head motion. The subjects were asked to keep their eyes closed and think of nothing in particular. The rsfMRI scans were performed using an echo-planar imaging (EPI) sequence: repetition time (TR)/echo time $(\mathrm{TE})=2000 \mathrm{~ms} / 40 \mathrm{~ms}$, flip angle $(\mathrm{FA})=90^{\circ}$, field of view $($ FOV $)=24 \times 24 \mathrm{~cm}$, matrix $=64 \times 64$, thickness $=4 \mathrm{~mm}$, gap $=1 \mathrm{~mm}$, voxel size $=3.75 \times 3.75 \times 4 \mathrm{~mm}^{3}$, bandwidth $=2232 \mathrm{~Hz} /$ pixel. Each volume comprised of 28 axial slices and 239 time points were collected. The scan lasted for $478 \mathrm{~s}$. The configuration data were collected by a 3D sagittal T1-weighted magnetization-prepared rapid gradient echo (MPRAGE) sequence: TR/TE $=1900 \mathrm{~ms} / 2.2 \mathrm{~ms}$, inversion time $(\mathrm{TI})=900 \mathrm{~ms}, \mathrm{FA}=9^{\circ}$, matrix $=256 \times 256$, slices $=176$, thickness $=1.0 \mathrm{~mm}$, no gap, voxel size $=1 \times 1 \times 1 \mathrm{~mm}^{3}$. All the subjects had not fallen asleep according to a simple questionnaire after the scan.

\section{Data Preprocessing}

Data preprocessing were performed using the Statistical Parametric Mapping $\left(\mathrm{SPM}^{1}\right)$ and Data Processing Assistant for rs-fMRI (DPARSF'; Chao-Gan and Yu-Feng, 2010) toolkits. Briefly, preprocessing included removal of the first 10 volumes, slice timing and head motion correction. To spatially normalize the fMRI data, the T1-weighted images were used to register the functional data to their corresponding anatomical image,

\footnotetext{
${ }^{1}$ www.fil.ion.ucl.ac.uk/spm

${ }^{2}$ www.restfmri.net/forum/DPARSF
}

and the resulting aligned $\mathrm{T} 1$ dataset was transformed into Montreal Neurological Institute (MNI) space (Ashburner and Friston, 2005). During the normalization, five subjects (two patients and three controls) were excluded. Then, the normalized functional images were created by transforming the T1 images to the customized T1 template. The inaccuracy of the spatial normalization of functional volumes caused by GM atrophy in the AD patients and HCs could be reduced by such a custom template-based registration procedure. The functional images were resampled to $3 \mathrm{~mm}$ isotropic voxels. Then these images were smoothed spatially (the full width-half maximum Gaussian kernel was set to $4 \mathrm{~mm}$ ). In order to reduce the influence of low-frequency drifts and high-frequency physiological noise, linear detrending and temporal bandpass filtering $(0.01-0.1 \mathrm{~Hz})$ were applied. Finally, several nuisance variables were removed by multiple linear regression analysis, including six head motion parameters, cerebrospinal fluid and white matter. During image preprocessing, no excessive head motion was found in our subjects (defined by a translation greater than $3 \mathrm{~mm}$ or rotation greater than $3^{\circ}$ ).

\section{Definition of Cerebellar Subregions}

The bilateral cerebellar subregions of Crus II and posterior lobule IX were extracted from the Probabilistic cerebellar atlas (Diedrichsen et al., 2009) with a threshold of $50 \%$ minimum probability (Figure 1).

\section{Functional Connectivity Analysis of the Cerebellar Subregions}

For each subject, the voxels of each cerebellar subregions were extracted and averaged to obtain the seed point reference time series. A correlation map was produced by computing the correlation coefficients between the reference time series and the time series from all the other brain voxels. Correlation coefficients were then converted to $z$ values using Fisher's $r$-to- $z$ transform to improve the normality. For all the subjects, four $z$-score maps were obtained which represented the intrinsic 
TABLE 2 | Regions showing AD-related resting state functional connectivity (rsFC) changes in cerebellar II and cerebellar IX.

\begin{tabular}{|c|c|c|c|c|c|c|}
\hline \multirow[t]{2}{*}{ ROls } & \multirow[t]{2}{*}{ Brain regions } & \multirow[t]{2}{*}{ Cluster voxels } & \multicolumn{3}{|c|}{ MNI coordinates (mm) } & \multirow[t]{2}{*}{ Maximum Z } \\
\hline & & & $x$ & $y$ & $z$ & \\
\hline \multirow[t]{2}{*}{ L. CB_II } & R.PHG & 55 & 24 & -45 & -3 & -4.83 \\
\hline & L.MOG & 49 & -21 & -93 & 0 & -4.68 \\
\hline R. CB_II & L.MOG & 89 & -21 & -93 & 0 & -4.87 \\
\hline \multirow[t]{2}{*}{ L. CB_IX } & R.PostCG & 53 & 66 & -3 & 21 & -4.38 \\
\hline & L.PreCG & 57 & -57 & 6 & 27 & -4.18 \\
\hline
\end{tabular}

Between groups differences were determined by two-sample $t$ tests $(P<0.001$, topoFDR corrected). Abbreviations: CB, cerebellar; $L$, left; MNI, Montreal Neurological Institute; MOG, middle occipital gyrus; PHG, parahippocampal gyrus; PostCG, postcentral gyrus; PreCG, precentral gyrus; $R$ right; Z, statistical value of peak voxel showing differences between two groups; $x, y, z$, coordinates of primary peak locations in the MNI space.

rsFCs of the four cerebellar subregions (two for each hemisphere).

\section{VBM Analysis}

Previous studies have reported significant atrophy of GM volume in the AD patients (Frisoni et al., 2002; Busatto et al., 2003). To eliminate the influences of GM atrophy on the rsFCs differences between the $\mathrm{AD}$ and $\mathrm{HC}$ group, we took voxel wise GM volume as covariate during the rsFC analysis ( $\mathrm{He}$ et al., 2007; Wang et al., 2011). First, each subject's GM volume map was estimated from the normalized T1 images by VBM method (VBM8 toolbox ${ }^{3}$; Ashburner and Friston, 2000). Then, we performed a two-sample $t$ test between the $\mathrm{AD}$ and $\mathrm{HC}$ group, this procedure was to show GM atrophy in the $\mathrm{AD}$ patients.

\section{Statistical Analysis}

To examine the within-group rsFCs of each cerebellar subregion for the $\mathrm{AD}$ patients and HCs, we performed one sample $t$ tests on individual $z$-score maps for each cerebellar subregion. The statistical significance threshold was set to $P<0.05$ with FWE corrected.

To assess the between-group differences of the whole-brain rsFCs of each cerebellar subregion, a two-sample $t$ tests was performed with age, gender and GM volume being treated as covariates. The significance threshold was set to $P<0.001$ with topo FDR corrected.

Then, partial correlation analysis was performed to explore the associations of the clinical variables with the rsFC strength

$\overline{{ }^{3} \text { http://dbm.neuro.uni-jena.de/vbm }}$

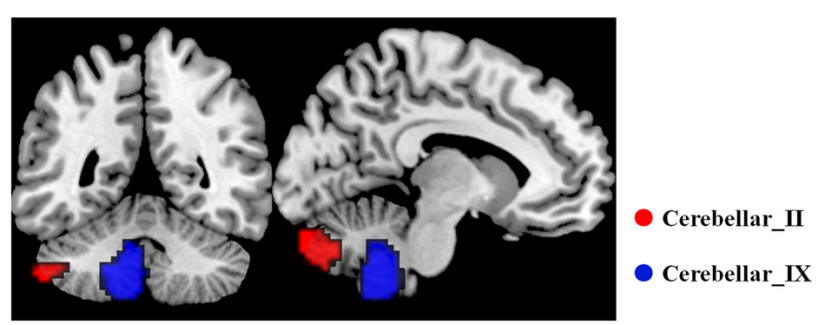

FIGURE 1| The subregions of cerebellar. In each hemisphere, two subregions were defined including the Crus II and posterior lobule IX using the probabilistic cerebellar atlas with a threshold of $50 \%$ minimum probability. Red color represents the Crus II, and green color represents the posterior lobule IX. for each cerebellar subregion in $\mathrm{AD}$ group, with age, gender as nuisance covariates $(P<0.05)$.

\section{RESULTS}

\section{Demographic and Neuropsychological Tests}

Table 1 showed the clinical information of all the participants. We did not find the significant differences of age and gender between the $\mathrm{AD}$ and $\mathrm{HC}$ group (both $P>0.1$ ). However, the AD group exhibited significantly lower MMSE, CDR, AVLT, ESD, MoCA, CDT, ADL and FAQ scores than the HC group $(P<0.0001)$

\section{Gray Matter Atrophy in the AD Group}

When comparing with the $\mathrm{HC}$ group, the $\mathrm{AD}$ group showed significant and widespread GM atrophy, which was mainly involved in the lateral temporal cortex, medial temporal lobe, dorsal and medial frontal cortices, posterior cingulate cortex and lateral parietal regions, as well as the subcortical regions (Figure 2).

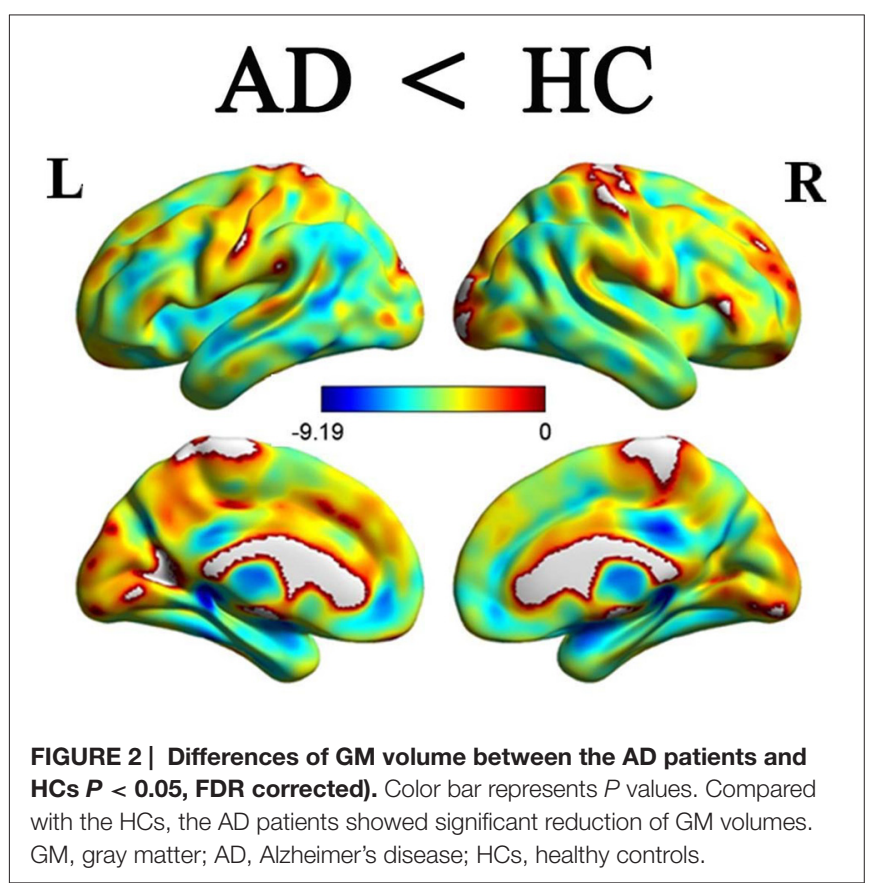



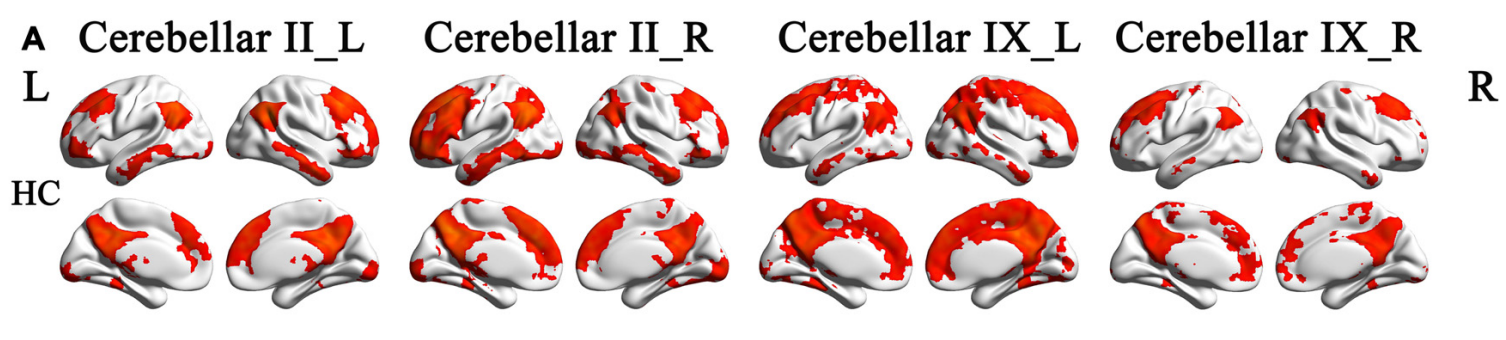

B
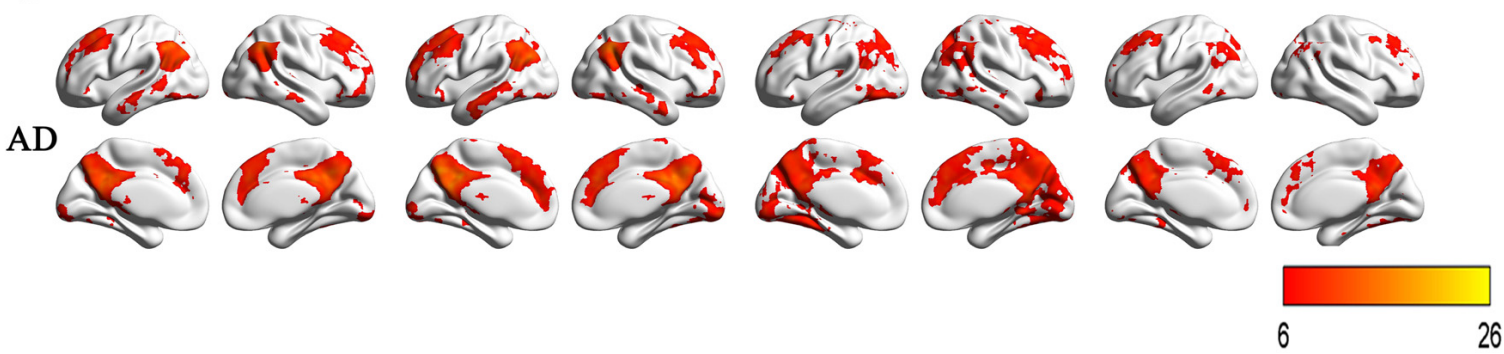

FIGURE 3 | The rsFCs patterns of the Crus II and posterior lobule IX in the HCs and AD patients. (A) The rsFCs patterns of the Crus II and posterior lobule IX in HCs ( $P<0.05$, FWE corrected); (B) the rsFCs patterns of the Crus II and posterior lobule IX in the AD patients ( $P<0.05$, FWE corrected). Color bar represent for positive functional connections. Similar patterns for each subregion between the two groups were shown by visual inspection. The cerebellar crus II was mainly correlated with the DMN regions, FPN regions and VN regions. The posterior lobule IX showed positive connectivity with the several frontal and parietal regions, including the DMN, FPN, VN regions and part of SMN regions. rsFC, resting state functional connectivity; HCs, healthy controls; AD, Alzheimer's disease; DMN, default-mode network; FPN, frontoparietal network; VN, visual network; SMN, sensorimotor network.

\section{Intragroup Functional Connectivity Maps of the Crus II and Posterior Lobule IX}

Figures 3A,B illustrated the rsFC maps for subregions of Crus II and posterior lobule IX within the HC and $\mathrm{AD}$ groups. By visual inspection, the two groups showed similar rsFC patterns for each cerebellar subregion. The cerebellar crus II was mainly correlated with the DMN regions including the posterior cingulate cortex/precuneus(PCC/Pcu), medial prefrontal cortex (MPFC), lateral parietal cortices and temporal cortices.
In addition, we also noticed the cerebellar crus II was associated with FPN regions including the dorsolateral prefrontal cortex (DLPFC) and posterior parietal cortex as well as visual network ( $\mathrm{VN})$ regions such as middle occipital region. The posterior lobule IX showed positive connectivity with the several frontal and parietal regions, including the DMN, FPN, $\mathrm{VN}$ regions and part of sensorimotor network (SMN) regions, including the bilateral precentral gyrus (PreCG) and postcentral gyrus (PostCG).
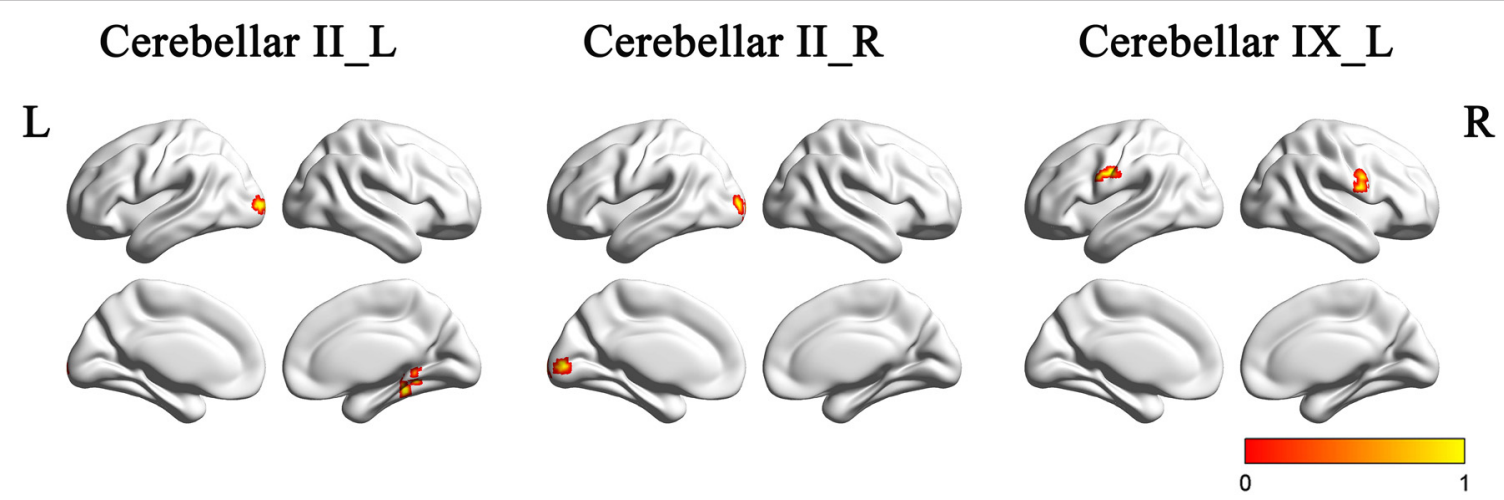

FIGURE 4 | The rsFCs differences between the AD patients and HCs $(\boldsymbol{P}<\mathbf{0 . 0 0 1}$, topoFDR corrected). Color bar indicates the frequency that the region emerged between group comparisons. Compared with the $\mathrm{HCs}$, the AD patients showed decreased rsFCs between the left Crus II and regions including the right PHG and left MOG, the right Crus II and the left MOG, the posterior lobule IX and SMN regions including PreCG and PostCG. rsFC, resting state functional connectivity; AD, Alzheimer's disease; HCs, healthy controls; PHG, parahippocampus; MOG, middle occipital gyrus; SMN, sensorimotor network; PreCG, precentral gyrus; PostCG, postcentral gyrus. 


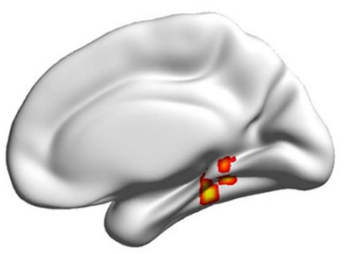

R.PHG

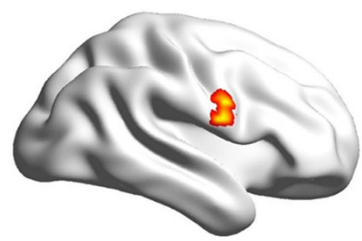

R.PostCG

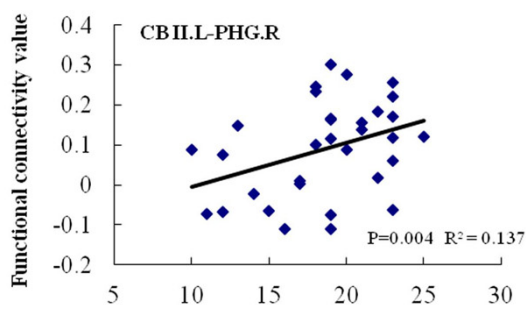

MMSE

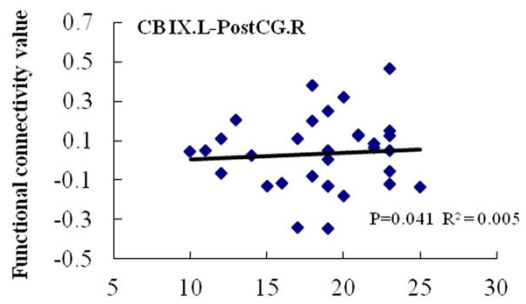

MMSE
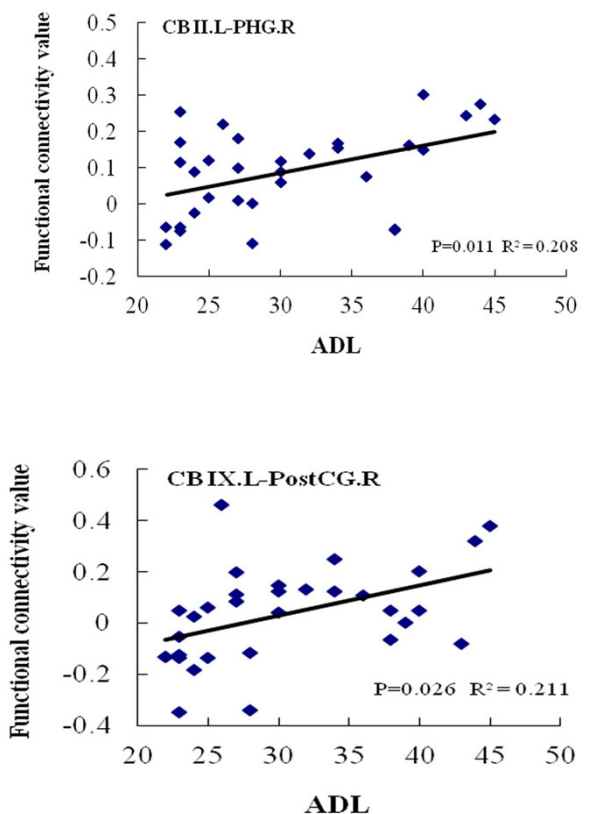

FIGURE 5 | Correlation of clinical variables and rsFCs of cerebellar subregions in the AD patients. MMSE scores showed positive correlations with the cerebellar subregional rsFCs including the left Crus II and the right PHG, the left lobule IX and right PostCG. In addition, ADL scores showed positive correlations with the cerebellar subregional rsFCs including the left Crus II and the right PHG, the left lobule IX and right PostCG. ADL, activities of daily living; L, left; MMSE, mini-mental state examination; MOG, middle occipital gyrus; PHG, parahippocampal gyrus; PostCG, postcentral gyrus; R, right; rsFC, resting-state functional connectivity.

\section{Intergroup Functional Connectivity Maps of the Crus II and Posterior Lobule IX}

Figure 4 and Table 2 showed the intergroup rsFC differences for each cerebellar subregion. Compared with the HC group, the AD group showed significantly decreased rsFCs between the left Crus II and regions including the right parahippocampus (PHG) and left middle occipital gyrus (MOG). In addition, the right Crus II showed significantly decreased rsFCs with the left MOG in the $\mathrm{AD}$ patients. When comparing the rsFC of the posterior lobule IX between the two groups, we found significantly decreased rsFCs in the $\mathrm{AD}$ patients in regions mainly located within the SMN regions including PreCG and PostCG. We didn't find significantly increased rsFCs of the regions in the $\mathrm{AD}$ patients comparing to the HCs.

\section{Correlations between the rsFCs of Cerebellar Subregions and Clinical Performances in the AD Patients}

In the $\mathrm{AD}$ group, positive correlations were found between the MMSE scores and the cerebellum subregional rsFC, including connectivity between the left Crus II and the right PHG, the left lobule IX and right PostCG. In addition, we found positive correlations between the ADL scores and rsFCs between the left Crus II and right PHG, the left lobule IX and right PostCG (Figure 5).

\section{DISCUSSION}

\section{Major Findings}

In the current study, we aim to investigate the intrinsic rsFCs of the cerebellar subregions in the $\mathrm{AD}$ patients by using rs-fMRI. By applying rsFC analysis to rs-fMRI data acquired from the $\mathrm{HCs}$ and $\mathrm{AD}$ patients, we observed two distinctive patterns of rsFCs for the cerebellar cognitive-related subregions: (1) the bilateral cerebellar Crus II exhibited rsFCs with the DMN, FPN and VN regions; and (2) the left lobule IX of the cerebellum was connected with the DMN, FPN, SMN and VN regions. Importantly, the $\mathrm{AD}$ patients showed differentially disrupted patterns of rsFCs in the specific subregions within different functional networks (DMN, VN and SMN), which were significantly associated with cognitive decline.

\section{Intragroup Functional Connectivity Maps of the Crus II and Posterior Lobule IX}

The Crus II of the cerebellum was functionally connected to several DMN regions (PCC/Pcu, MPFC, IPL, lateral parietal cortices and temporal cortices) and FPN regions (DLPFC and posterior parietal cortex). In the previous studies, Krienen and Buckner (2009) demonstrated Crus II played an important role in DMN. Some other studies revealed that the Crus II was typically connected with the FPN regions (Sang et al., 2012; Li et al., 2013). In the current study, 
the crus II was mainly connected with both the DMN and FPN regions, which was consistent with the previous studies.

The lobule IX of the cerebellum was involved in the DMN, FPN, VN and SMN regions. Several previous studies (Habas et al., 2009; Sang et al., 2012; Li et al., 2013) reported the lobule IX was linked with the DMN regions, which was similar with the current study. In addition to the DMN regions, the present study also found that the lobule IX was connected with the FPN, SMN and VN regions.

\section{Intergroup Functional Connectivity Maps of the Crus II and Posterior Lobule IX}

We found that the subregions of the cerebellum showed differentially disrupted rsFCs in the AD patients. Specifically, we observed disrupted rsFCs between the Crus II and the right PHG, bilateral MOG. In addition, we also found the decreased rsFCs between the posterior lobule IX and the SMN regions (PreCG and PostCG).

PHG is the critical node of the DMN. Several studies have reported the cortical thinning (Dickerson and Sperling, 2009), disrupted functional activity and connectivity (Greicius et al., 2004; Wang et al., 2011) of the DMN regions in the AD patients. Based on the previous findings, our findings provided further evidence for the destruction of the DMN in the AD patients, which was especially relevant to the disconnection between the Crus II of cerebellum and PHG. More importantly, we found that the rsFC values between the left Crus II and the right PHG were positively correlated with MMSE and ADL scores, suggesting the clinical relevance of functional disconnection in the AD patients.

Besides the PHG region, we also observed disrupted connectivity between the bilateral Crus II and the left MOG. The MOG is located in the primary visual cortex and play a critical role in visual cognition. By using fMRI method, Sala-Llonch et al. (2015) reported MOG region presented higher activity during the task of visuo-perceptual working memory. Using diffusion tensor imaging (DTI) and tractography, a previous study demonstrated that the structural disconnection in ventral occipital-temporal cortex contributed to the deficit in facial recognition (Thomas et al., 2009). Visual cognition deficits were consistently reported to accompany the development of AD (Cronin-Golomb, 1995; Bokde et al., 2006).

For the disrupted rsFC of SMN in the AD patients, some task-related $\mathrm{fMRI}$ studies have demonstrated reduced activation in the premotor cortex in the $\mathrm{AD}$ patients when performing the motor-related tasks (Agosta et al., 2010; Vidoni et al., 2012). Brier et al. (2012) found the significant functional abnormality of SMN in the $\mathrm{AD}$ patients by analyzing rs-fMRI data of 510 human subjects. In conjunction with these findings, we speculated that the $\mathrm{AD}$ patients might presented subtle motor impairment, which caused by the dysfunction of the SMN.

Pathologically, understanding the contribution of tau and amyloid- $\beta$ pathology to cerebellum in $\mathrm{AD}$ would further expand our knowledge of the disease mechanisms (Liu et al., 2012). In some studies, the cerebellum was typically devoid of amyloid plaques and tau (Serrano-Pozo et al., 2011; Ni et al., 2013).
While other studies revealed deposits of amyloid- $\beta$ plaques, neurofibrillary tangles in the cerebellum in the $\mathrm{AD}$ patients (Larner, 1997; Ciavardelli et al., 2010; Sepulveda-Falla et al., 2011). Previous studies confirmed the majority of the cerebellum is connected to cerebral association networks (Buckner, 2013). Furthermore, our study revealed the disconnection between cerebellar sub-regions and several cerebral networks in the $\mathrm{AD}$ patients. We speculated that the disrupted rsFCs of the cerebellum in $\mathrm{AD}$ might be due to the pathological changes in the cerebellum. Further studies need to be performed in the future.

\section{Analysis of Age, Gender and GM Volume}

In the current study, several factors including age, gender and GM volume would affect the final results. In collecting subjects, we paid high attention to these factors to match the two groups as well as possible. In the following rsFCs analysis, we used age, gender and GM volume as covariates to avoid the impact of the factors. As the results, we found the rsFCs changes of cerebellum in $\mathrm{AD}$ were independent of the above factors.

\section{Future Consideration}

Several issues need to be considered in the future. First, in the current study, we focused on the rsFCs of the cerebellar sub-regions only by using the rsfMRI. Further studies that simultaneously combine the fMRI and DTI methods on the cerebellum would provide more information for the mechanism of $\mathrm{AD}$. Second, to explore the early stage of $\mathrm{AD}$ patients would be helpful to find the valuable biomarker for diagnosis of the disease. For example, amnestic mild cognitive impairments (aMCI) and ApoE-4 allele carriers were paid more attention in the recent studies (Pievani et al., 2011b; Bai et al., 2012). Finally, to clarify the progressive changes of the connectivity in cerebellum subregions, a longitudinal research would be essential, which may provide a deep understanding of the $\mathrm{AD}$ pathophysiological mechanisms.

\section{CONCLUSION}

We have identified the patterns of abnormal rsFCs in the two cognitive-related cerebellar subregions in the AD patients, which was involved in different functional brain networks such as DMN, VN and SMN. These findings added the new evidence for the disconnection syndrome of the disease, which might be helpful to search for the potential biomarker for early $\mathrm{AD}$ diagnosis in the future.

\section{AUTHOR CONTRIBUTIONS}

WZ and XL: the conception or design of the work; the acquisition, analysis and the interpretation of data for the work; drafting the work; final approval of the version to be published; agreement to be accountable for all aspects of the work; HS: the acquisition of data for the work; clinical evaluation; KL and ZW: the design of the work; revising the work; final approval of the version to be published; agreement to be accountable for all aspects of the work. 


\section{FUNDING}

This work was supported by the National Natural Scientific Foundation of China (Grant Nos. 81370037, 81571648 and 81471649).

\section{REFERENCES}

Agosta, F., Rocca, M. A., Pagani, E., Absinta, M., Magnani, G., Marcone, A., et al. (2010). Sensorimotor network rewiring in mild cognitive impairment and Alzheimer's disease. Hum. Brain Mapp. 31, 515-525. doi: 10.1002/hbm.20883

Ashburner, J., and Friston, K. J. (2000). Voxel-based morphometry-the methods. Neuroimage 11, 805-821. doi: 10.1006/nimg.2000.0582

Ashburner, J., and Friston, K. J. (2005). Unified segmentation. Neuroimage 26, 839-851. doi: 10.1016/j.neuroimage.2005.02.018

Bai, F., Liao, W., Watson, D. R., Shi, Y., Yuan, Y., Cohen, A. D., et al. (2011). Mapping the altered patterns of cerebellar resting-state function in longitudinal amnestic mild cognitive impairment patients. J. Alzheimers Dis. 23, 87-99. doi: 10.3233/JAD-2010-101533

Bai, F., Shu, N., Yuan, Y., Shi, Y., Yu, H., Wu, D., et al. (2012). Topologically convergent and divergent structural connectivity patterns between patients with remitted geriatric depression and amnestic mild cognitive impairment. J. Neurosci. 32, 4307-4318. doi: 10.1523/JNEUROSCI. 5061-11.2012

Bas, O., Acer, N., Mas, N., Karabekir, H. S., Kusbeci, O. Y., and Sahin, B. (2009). Stereological evaluation of the volume and volume fraction of intracranial structures in magnetic resonance images of patients with Alzheimer's disease. Ann. Anat. 191, 186-195. doi: 10.1016/j.aanat.2008.12.003

Biswal, B., Yetkin, F. Z., Haughton, V. M., and Hyde, J. S. (1995). Functional connectivity in the motor cortex of resting human brain using echo-planar MRI. Magn. Reson. Med. 34, 537-541. doi: 10.1002/mrm.1910340409

Bokde, A. L., Lopez-Bayo, P., Meindl, T., Pechler, S., Born, C., Faltraco, F., et al. (2006). Functional connectivity of the fusiform gyrus during a face-matching task in subjects with mild cognitive impairment. Brain 129, 1113-1124. doi: 10.1093/brain/awl051

Braak, H., and Braak, E. (1991). Neuropathological stageing of Alzheimer-related changes. Acta Neuropathol. 82, 239-259. doi: 10.1007/bf00308809

Brier, M. R., Thomas, J. B., Snyder, A. Z., Benzinger, T. L., Zhang, D., Raichle, M. E., et al. (2012). Loss of intranetwork and internetwork resting state functional connections with Alzheimer's disease progression. J. Neurosci. 32, 8890-8899. doi: 10.1523/JNEUROSCI.5698-11.2012

Buckner, R. L. (2013). The cerebellum and cognitive function: 25 years of insight from anatomy and neuroimaging. Neuron 80, 807-815. doi: 10.1016/j.neuron. 2013.10.044

Buckner, R. L., Sepulcre, J., Talukdar, T., Krienen, F. M., Liu, H., Hedden, T., et al. (2009). Cortical hubs revealed by intrinsic functional connectivity: mapping, assessment of stability and relation to Alzheimer's disease. J. Neurosci. 29, 1860-1873. doi: 10.1523/JNEUROSCI.5062-08.2009

Busatto, G. F., Garrido, G. E., Almeida, O. P., Castro, C. C., Camargo, C. H., Cid, C. G., et al. (2003). A voxel-based morphometry study of temporal lobe gray matter reductions in Alzheimer's disease. Neurobiol. Aging 24, 221-231. doi: 10.1016/s0197-4580(02)00084-2

Castellazzi, G., Palesi, F., Casali, S., Vitali, P., Sinforiani, E., WheelerKingshott, C. A., et al. (2014). A comprehensive assessment of resting state networks: bidirectional modification of functional integrity in cerebrocerebellar networks in dementia. Front. Neurosci. 8:223. doi: 10.3389/fnins. 2014.00223

Chao-Gan, Y., and Yu-Feng, Z. (2010). DPARSF: a MATLAB toolbox for "Pipeline" data analysis of resting-state fMRI. Front. Syst. Neurosci. 4:13. doi: $10.3389 /$ fnsys.2010.00013

Chételat, G., Desgranges, B., Landeau, B., Mézenge, F., Poline, J. B., De la Sayette, V., et al. (2008). Direct voxel-based comparison between grey matter hypometabolism and atrophy in Alzheimer's disease. Brain 131, 60-71. doi: 10.1093/brain/awm288

Ciavardelli, D., Silvestri, E., Del Viscovo, A., Bomba, M., De Gregorio, D., Moreno, M., et al. (2010). Alterations of brain and cerebellar proteomes

\section{ACKNOWLEDGMENTS}

The authors thank $\mathrm{AD}$ research team for their hard work on collecting subject data, and all the subjects who participated in the study.

linked to $A \beta$ and tau pathology in a female triple-transgenic murine model of Alzheimer's disease. Cell Death Dis. 1:e90. doi: 10.1038/cddis.2010.68

Cronin-Golomb, A. (1995). Vision in Alzheimer's disease. Gerontologist 35, 370-376.

Dai, Z., Yan, C., Wang, Z., Wang, J., Xia, M., Li, K., et al. (2012). Discriminative analysis of early Alzheimer's disease using multi-modal imaging and multilevel characterization with multi-classifier (M3). Neuroimage 59, 2187-2195. doi: 10.1016/j.neuroimage.2011.10.003

Dickerson, B. C., and Sperling, R. A. (2009). Large-scale functional brain network abnormalities in Alzheimer's disease: insights from functional neuroimaging. Behav. Neurol. 21, 63-75. doi: 10.3233/BEN-2009-0227

Diedrichsen, J., Balsters, J. H., Flavell, J., Cussans, E., and Ramnani, N. (2009). A probabilistic MR atlas of the human cerebellum. Neuroimage 46, 39-46. doi: 10.1016/j.neuroimage.2009.01.045

Dubois, B., Feldman, H. H., Jacova, C., Cummings, J. L., Dekosky, S. T., Barberger-Gateau, P., et al. (2010). Revising the definition of Alzheimer's disease: a new lexicon. Lancet Neurol. 9, 1118-1127. doi: 10.1016/S14744422(10)70223-4

Dubois, B., Feldman, H. H., Jacova, C., Dekosky, S. T., Barberger-Gateau, P., Cummings, J., et al. (2007). Research criteria for the diagnosis of Alzheimer's disease: revising the NINCDS-ADRDA criteria. Lancet Neurol. 6, 734-746. doi: 10.1016/s1474-4422(07)70178-3

Fox, M. D., and Raichle, M. E. (2007). Spontaneous fluctuations in brain activity observed with functional magnetic resonance imaging. Nat. Rev. Neurosci. 8, 700-711. doi: $10.1038 / \mathrm{nrn} 2201$

Frisoni, G. B., Testa, C., Zorzan, A., Sabattoli, F., Beltramello, A., Soininen, H., et al. (2002). Detection of grey matter loss in mild Alzheimer's disease with voxel based morphometry. J. Neurol. Neurosurg. Psychiatry 73, 657-664. doi: 10.1136/jnnp.73.6.657

Greicius, M. D., Srivastava, G., Reiss, A. L., and Menon, V. (2004). Defaultmode network activity distinguishes Alzheimer's disease from healthy aging: evidence from functional MRI. Proc. Natl. Acad. Sci. U S A 101, 4637-4642. doi: 10.1073/pnas.0308627101

Habas, C., Kamdar, N., Nguyen, D., Prater, K., Beckmann, C. F., Menon, V., et al. (2009). Distinct cerebellar contributions to intrinsic connectivity networks. J. Neurosci. 29, 8586-8594. doi: 10.1523/JNEUROSCI.1868-09.2009

Hauser, T., Schönknecht, P., Thomann, P. A., Gerigk, L., Schröder, J., Henze, R., et al. (2013). Regional cerebral perfusion alterations in patients with mild cognitive impairment and Alzheimer disease using dynamic susceptibility contrast MRI. Acad. Radiol. 20, 705-711. doi: 10.1016/j.acra.2013. 01.020

He, Y., Wang, L., Zang, Y., Tian, L., Zhang, X., Li, K., et al. (2007). Regional coherence changes in the early stages of Alzheimer's disease: a combined structural and resting-state functional MRI study. Neuroimage 35, 488-500. doi: 10.1016/j.neuroimage.2006.11.042

Kandimalla, R. J., Anand, R., Veeramanikandan, R., Wani, W. Y., Prabhakar, S., Grover, V. K., et al. (2014). CSF ubiquitin as a specific biomarker in Alzheimer's disease. Curr. Alzheimer Res. 11, 340-348. doi: $10.2174 / 1567205011666140331161027$

Kandimalla, R. J., Prabhakar, S., Binukumar, B. K., Wani, W. Y., Gupta, N., Sharma, D. R., et al. (2011). Apo-E\&4 allele in conjunction with A $\beta 42$ and tau in CSF: biomarker for Alzheimer's disease. Curr. Alzheimer Res. 8, 187-196. doi: $10.2174 / 156720511795256071$

Kaufmann, L., Ischebeck, A., Weiss, E., Koppelstaetter, F., Siedentopf, C., Vogel, S. E., et al. (2008). An fMRI study of the numerical stroop task in individuals with and without minimal cognitive impairment. Cortex 44, 1248-1255. doi: 10.1016/j.cortex.2007.11.009

Krienen, F. M., and Buckner, R. L. (2009). Segregated fronto-cerebellar circuits revealed by intrinsic functional connectivity. Cereb. Cortex 19, 2485-2497. doi: 10.1093/cercor/bhp135 
Larner, A. J. (1997). The cerebellum in Alzheimer's disease. Dement. Geriatr. Cogn. Disord. 8, 203-209. doi: 10.1159/000106632

Li, W., Han, T., Qin, W., Zhang, J., Liu, H., Li, Y., et al. (2013). Altered functional connectivity of cognitive-related cerebellar subregions in well-recovered stroke patients. Neural Plast. 2013:452439. doi: 10.1155/2013/452439

Liu, L., Drouet, V., Wu, J. W., Witter, M. P., Small, S. A., Clelland, C., et al. (2012). Trans-synaptic spread of tau pathology in vivo. PLoS One 7:e31302. doi: 10.1371/journal.pone.0031302

Mevel, K., Desgranges, B., Baron, J. C., Landeau, B., De la Sayette, V., Viader, F., et al. (2007). Detecting hippocampal hypometabolism in mild cognitive impairment using automatic voxel-based approaches. Neuroimage 37, 18-25. doi: 10.1016/j.neuroimage.2007.04.048

Ni, R., Gillberg, P. G., Bergfors, A., Marutle, A., and Nordberg, A. (2013). Amyloid tracers detect multiple binding sites in Alzheimer's disease brain tissue. Brain 136, 2217-2227. doi: 10.1093/brain/awt142

O'Reilly, J. X., Beckmann, C. F., Tomassini, V., Ramnani, N., and JohansenBerg, H. (2010). Distinct and overlapping functional zones in thecerebellum defined by resting state functional connectivity. Cereb. Cortex 20, 953-965. doi: 10.1093/cercor/bhp157

Pievani, M., de Haan, W., Wu, T., Seeley, W. W., and Frisoni, G. B. (2011a). Functional network disruption in the degenerative dementias. Lancet Neurol. 10, 829-843. doi: 10.1016/s1474-4422(11)70158-2

Pievani, M., Galluzzi, S., Thompson, P. M., Rasser, P. E., Bonetti, M., and Frisoni, G. B. (2011b). APOE4 is associated with greater atrophy of the hippocampal formation in Alzheimer's disease. Neuroimage 55, 909-919. doi: 10.1016/j.neuroimage.2010.12.081

Sala-Llonch, R., Palacios, E. M., Junqué, C., Bargalló, N., and Vendrell, P. (2015). Functional networks and structural connectivity of visuospatial and visuoperceptual working memory. Front. Hum. Neurosci. 9:340. doi: $10.3389 /$ fnhum. 2015.00340

Sang, L., Qin, W., Liu, Y., Han, W., Zhang, Y., Jiang, T., et al. (2012). Restingstate functional connectivity of the vermal and hemispheric subregions of the cerebellum with both the cerebral cortical networks and subcortical structures. Neuroimage 61, 1213-1225. doi: 10.1016/j.neuroimage.2012. 04.011

Schmahmann, J. D., Weilburg, J., and Sherman, J. C. (2007). The neuropsychiatry of the cerebellum-insights from the clinic. Cerebellum 6, 254-267. doi: $10.1080 / 14734220701490995$

Seeley, W. W., Crawford, R. K., Zhou, J., Miller, B. L., and Greicius, M. D. (2009). Neurodegenerative diseases target large-scale human brain networks. Neuron 62, 42-52. doi: 10.1016/j.neuron.2009.03.024

Sepulveda-Falla, D., Matschke, J., Bernreuther, C., Hagel, C., Puig, B., Villegas, A., et al. (2011). Deposition of hyperphosphorylated tau in cerebellum of PS1 E280A Alzheimer's disease. Brain Pathol. 21, 452-463. doi: 10.1111/j.17503639.2010.00469.x

Serrano-Pozo, A., Frosch, M. P., Masliah, E., and Hyman, B. T. (2011). Neuropathological alterations in Alzheimer disease. Cold Spring Harb. Perspect. Med. 1:a006189. doi: 10.1101/cshperspect.a006189

Stoodley, C. J., and Schmahmann, J. D. (2009). Functional topography in the human cerebellum: a meta-analysis of neuroimaging studies. Neuroimage 44, 489-501. doi: 10.1016/j.neuroimage.2008.08.039

Strata, P. (2015). The emotional cerebellum. Cerebellum 14, 570-577. doi: $10.1007 / \mathrm{s} 12311-015-0649-9$
Thomann, P. A., Schläfer, C., Seidl, U., Santos, V. D., Essig, M., and Schröder, J. (2008). The cerebellum in mild cognitive impairment and Alzheimer's disease-a structural MRI study. J. Psychiatr. Res. 42, 1198-1202. doi: 10.1016/j. jpsychires.2007.12.002.

Thomas, C., Avidan, G., Humphreys, K., Jung, K. J., Gao, F., and Behrmann, M. (2009). Reduced structural connectivity in ventral visual cortex in congenital prosopagnosia. Nat. Neurosci. 12, 29-31. doi: $10.1038 / \mathrm{nn} .2224$

Vidoni, E. D., Thomas, G. P., Honea, R. A., Loskutova, N., and Burns, J. M. (2012). Evidence of altered corticomotor system connectivity in early-stage Alzheimer's disease. J. Neurol. Phys. Ther. 36, 8-16. doi: 10.1097/NPT. 0b013e3182462ea6

Wang, Z., Jia, X., Liang, P., Qi, Z., Yang, Y., Zhou, W., et al. (2012). Changes in thalamus connectivity in mild cognitive impairment: evidence from resting state fMRI. Eur. J. Radiol. 81, 277-285. doi: 10.1016/j.ejrad.2010. 12.044

Wang, K., Liang, M., Wang, L., Tian, L., Zhang, X., Li, K., et al. (2007). Altered functional connectivity in early Alzheimer's disease: a restingstate fMRI study. Hum. Brain Mapp. 28, 967-978. doi: 10.1002/hbm. 20324

Wang, Z., Xia, M., Dai, Z., Liang, X., Song, H., He, Y., et al. (2015). Differentially disrupted functional connectivity of the subregions of the inferior parietal lobule in Alzheimer's disease. Brain Struct. Funct. 220, 745-762. doi: 10.1007/s00429-013-0681-9

Wang, Z., Yan, C., Zhao, C., Qi, Z., Zhou, W., Lu, J., et al. (2011). Spatial patterns of intrinsic brain activity in mild cognitive impairment and Alzheimer's disease: a resting-state functional MRI study. Hum. Brain Mapp. 32, 1720-1740. doi: $10.1002 / \mathrm{hbm} .21140$

Wang, L., Zang, Y., He, Y., Liang, M., Zhang, X., Tian, L., et al. (2006). Changes in hippocampal connectivity in the early stages of Alzheimer's disease: evidence from resting state fMRI. Neuroimage 31, 496-504. doi: 10.1016/j.neuroimage. 2005.12.033

Zhang, D., and Raichle, M. E. (2010). Disease and the brain's dark energy. Nat. Rev. Neurol. 6, 15-28. doi: 10.1038/nrneurol.2009.198

Zhang, Z., Liu, Y., Zhou, B., Zheng, J., Yao, H., An, N., et al. (2014). Altered functional connectivity of the marginal division in Alzheimer's disease. Curr. Alzheimer Res. 11, 145-155. doi: 10.2174/15672050116661401101 12608

Zhang, H. Y., Wang, S. J., Liu, B., Ma, Z. L., Yang, M., Zhang, Z. J., et al. (2010). Resting brain connectivity: changes during the progress of Alzheimer disease. Radiology 256, 598-606. doi: 10.1148/radiol.10091701

Conflict of Interest Statement: The authors declare that the research was conducted in the absence of any commercial or financial relationships that could be construed as a potential conflict of interest.

Copyright $\odot 2017$ Zheng, Liu, Song, Li and Wang. This is an open-access article distributed under the terms of the Creative Commons Attribution License (CC BY). The use, distribution or reproduction in other forums is permitted, provided the original author(s) or licensor are credited and that the original publication in this journal is cited, in accordance with accepted academic practice. No use, distribution or reproduction is permitted which does not comply with these terms. 